\title{
Mine de plomb (Les Géorgiques, II)
}

\section{Olivier Rolin}

\section{(2) OpenEdition}

\section{Journals}

Édition électronique

URL : http://journals.openedition.org/ccs/504

DOI : $10.4000 / \operatorname{ccs} 504$

ISSN : 2558-782X

\section{Éditeur :}

Presses universitaires de Rennes, Association des lecteurs de Claude Simon

\section{Édition imprimée}

Date de publication : 31 décembre 2006

Pagination : 121-126

ISBN : 9782914518895

ISSN : 1774-9425

\section{Référence électronique}

Olivier Rolin, « Mine de plomb (Les Géorgiques, II) », Cahiers Claude Simon [En ligne], 2 | 2006, mis en ligne le 20 septembre 2017, consulté le 15 septembre 2020. URL : http://journals.openedition.org/ccs/ 504 


\section{Mine de plomb \\ (Les Géorgiques, II) \\ par Olivier ROLIN}

Récemment, la revue Le Nouveau Recueil m’a demandé, à moi parmi d'autres, de réfléchir autour d'une phrase de Flaubert dans une lettre à Tourgueniev, qui évoque la " beauté » à laquelle on peut atteindre dans la langue française, et qui devrait être le souci de tout écrivain. J'ai essayé, mais je crains de n'être pas parvenu à dire grand-chose de pertinent sur la beauté des mots, de la phrase. Cet hommage à Claude Simon me sera l'occasion d'y revenir (et sans doute d'y échouer derechef). Je voudrais tenter de dire, modestement, laborieusement, en quoi la phrase de Claude Simon est belle - car il y a peu de phrase, dans la littérature française moderne, qui fasse aussi profonde impression de beauté, je dirai même (bien que le mot soit "passé de mode ", mais Claude Simon est heureusement "passé de mode ", outrepassant de très loin toute mode, dans un espace et un temps où se trouvent aussi Tacite et Shakespeare) : de grandeur. Je le ferai en me souvenant d'une lecture publique que $j$ 'ai faite il y a un an d'un passage des Géorgiques, le début de la partie II. Je me souviens avoir été littéralement transporté, enthousiasmé je prends ce mot au sens fort, grec, de possession par un dieu. Je me souviens avoir trouvé qu'en dépit des apparences ce n'était pas difflcile de lire Claude Simon, qu'il y avait dans sa phrase quelque chose, 
une puissance, une précision, un rythme qui demandaient à éclater en sons, à être proférés, qu'il suffisait en quelque sorte de se plier à cette impérieuse injonction, de se laisser traverser par la force des mots. Un peu d'alcool contribuait peut-être à cette découverte - il était très tard, c'était la " Nuit blanche " dans la librairie "Les Cahiers de Colette ", à Paris -, néanmoins des relectures parfaitement sobres ne m'ont pas fait changer d'avis. L'alcool n'a pas làdedans plus d'importance que les feuilles de laurier mâchées par la Pythie.

Les soldats sont dans un train, dans un wagon à bestiaux. Ils ne savent pas où ils vont. Le froid oblige à maintenir fermées les portes coulissantes. En collant l'œil à l'interstice qui sépare les planches des parois, on aperçoit une campagne enneigée, sur quoi tombe la nuit. Chocs saccadés des roues aux jointures des rails. Le train s'arrête enfin. On saute sur le ballast, on fait descendre, d'autres wagons, les chevaux. Cependant que se forment les pelotons, passe en tonnerre un train de voyageurs. On saute en selle, la marche commence, sans but, "dans l'hiver et dans la nuit ", comme dit la chanson des Gardes Suisses placée en exergue du Voyage au bout de la nuit (et d'ailleurs il semble rouler dans ces pages un écho lointain de la marche nocturne du cavalier Bardamu vers Noirceur-sur-la-Lys). Bientôt, la fatigue, le découragement, l'obscurité, la neige qui tombe font que l'escadron se disloque. Voilà ce que peignent, ce que dessinent, plutôt, à la mine de plomb, les pages du début de la section II des Géorgiques. Je n'emploie pas par hasard, bien sûr, cette expression de "dessin à la mine de plomb " : c'est celle qu'utilise Claude Simon pour suggérer la netteté minutieuse de la vision : "Ils perçoivent cela d'un coup et pourtant de façon détaillée (ou plutôt dénudée, fouillée, comme un de ces dessins minutieux et précis à la mine de plomb) ${ }^{1}$ ». Cela : le ciel et la ligne fuyante des rails, le train, la plaine enneigée hachurée de boqueteaux, les trémies rouillées d'une ancienne sablière, peut-être, un fossé d'eau stagnante, des fourrés de ronce, et pas seulement les ronces mais les « paquets de neige molle

\footnotetext{
${ }^{1}$ Claude Simon, Les Géorgiques, Minuit, 1981, p. 85 (abrégé désormais en G).
} 
accrochés dans leurs enchevêtrements » $(G, 85)$, pas seulement le fossé mais "les minces triangles de glace, comme du verre dépoli, sale et grisâtre à la surface de l'eau noire ", pas seulement les trémies rouillées mais la peinture boursouflée, effritée, formant comme une cicatrice rougeâtre sur le pourtour des plaques de rouille.

De cette acuité extrême du regard, qui distingue tout et n'exclut rien, de cette précision scrupuleuse, fourmillante, du trait, vient une partie du prodigieux " effet de réel " de l'écriture de Claude Simon. Rien de moins " intellectuel ", ou plutôt (formulation adverbiale éminemment simonienne, dénotant l'effort constant pour cerner, serrer le réel d'aussi près que le permettent les mots), rien de moins abstrait, rien de plus matériel, de plus adepte du "parti pris des choses ». C'est le sens, bien sûr (là, je reprends des remarques faites par Lucien Dällenbach dans la monographie qu'il lui a consacrée), de sa défense de Dürer contre Élie Faure, dans La Bataille de Pharsale. "Tout est au même plan dans la nature ${ }^{2}$ ", voilà ce qu'Élie Faure reproche à l'artiste allemand (phrase qui me fait penser au vers de Whitman : I believe a leaf of grass is no less than the journey-work of the stars, "Je crois qu'une feuille d'herbe ne vaut pas moins que le travail errant des étoiles »). Eh bien oui : tout est immanent, tout est là, tout est vu et doit être dit "d'un coup et pourtant de façon détaillée ». La " mine de plomb» de Claude Simon embrasse, dans un seul mouvement, à la fois très ample et très réticulé, l'espace et ce qui le remplit, elle joue vertigineusement du panoramique et du zoom, elle montre la forêt, sa masse, sa rumeur, et la fine articulation de la feuille sur sa tige, l'armée en déroute et le poil luisant de sueur sur la croupe d'un cheval, elle va sans cesse du cosmos à la chose infime, et c'est ce battement en elle qui lui confere je crois cette puissance un peu grisante qui impose au lecteur sa loi. Elle a quelque chose de l'aleph borgésien : "L'espace cosmique était là, sans diminution de volume ".

2 Claude Simon, La Bataille de Pharsale, Minuit 1969. 
Ce que voient les cavaliers qui s'ébranlent : des pavillons ouvriers, quelques fenêtres allumées, des jardinets, des choux aux feuilles brûlées par le gel, flasques "sous leurs chapeaux de neige (et même pas assez de neige pour tout recouvrir, pour cacher les tiges annelées et pourries, les sommets des sillons, la terre noirâtre) " $(G, 89)$, un fourbi de pauvres choses éparses, et en même temps la plaine " blanche ou plutôt gris blanc " (90), la mince ligne faiblement lumineuse où se joignent et se confondent presque à l'horizon les étendues désertes de la plaine et du ciel. Le froid est une chose "pour ainsi dire cosmique "(102), une matière vitreuse serrée comme par une presse "au-dessus des forêts, des collines, des rares fermes éparses dans la campagne blanche ", mais qui, s'insinuant "dans les narines, la bouche, les poumons ", finit par envahir «le corps selon les lacis compliqués des bronches, des bronchioles, des vaisseaux, se divisant, se ramifiant, poussant des radicelles épineuses dans chacun des membres, des doigts, des orteils " : et ce mouvement, qui va du cosmos jusque dans les plus intimes canaux du corps, est une métaphore du mouvement qu'accomplit la phrase de Claude Simon. Je la comparerais volontiers aussi à la progression d'une marée montante, chacun a vu cela aller sur le sable, immense et délicat, irrésistible et pointilleux, avançant selon un front, ployant de côté et d'autres des filets d'eau rapide, investissant, cernant, noyant inexorablement chaque petite éminence.

Et cette phrase pour ainsi dire fractale est capable de dire non seulement le fourmillement de ce qui s'offre simultanément au regard, mais aussi la succession, la trajectoire que le temps creuse dans le donné. Non seulement l'instantané, mais aussi le mouvement, l'apparition et la disparition. Plasticité admirable de la matière verbale, photographique et cinématographique à la fois. Descendus sur le ballast, dans le crépuscule, les cavaliers voient passer un express de voyageurs, comme une image d'un monde qu'ils ont quitté. C'est d'abord le bruit annonciateur, sans rien de visible encore, puis un point, qui grossit, se transforme en une ligne filant à toute vitesse de wagons verdâtres aux fenêtres desquelles paraît une humanité étrange, demeurée du côté de la paix, de la quiétude, 
femme donnant le biberon, petite fille avec un nœud dans les cheveux, homme en manches de chemise, puis l'apparition se résorbe, se rétracte, jusqu'à n'être plus qu'une lanterne rouge dans l'ombre, puis un point, puis plus rien, une fumée, une odeur de charbon qui un instant encore flotte avant de se disperser, tout cela, cette vision s'ouvrant, se déployant puis se refermant au long d'une phrase dont le grondement croissant puis décroissant est rythmé par le martèlement des roues aux cassures des rails, une phrase à la perspective aussi vertigineuse que celle d'affiches célèbres de Cassandre. Et la même cinématographie, quelque vingt-cinq pages plus loin, les lumignons des vélos s'allumant dans la nuit, à la sortie des aciéries, s'approchant, s'arrêtant au poste de garde, repartant, s'éloignant, « la lumière du fanal reflétée faiblement par les derniers feux arrière qui jetaient dans les ténèbres glaciales de fugitifs éclats de rubis " $(G, 114)$, tout ce mouvement orchestré par le craquement de la neige, des tintements de métal, le sifflement des freins...

S'il n'est pas difficile de lire, à haute voix, Claude Simon (en tout cas : pas si difficile qu'on le croit, que cela parait d'abord), si c'est une expérience, au sens que j'ai dit, enthousiasmante, c'est parce que ses phrases sont comme saturées d'une puissance matérielle, d'un pouvoir d'évocation physique qui impose presque aux mots écrits de devenir des mots incarnés, proférés dans l'air physique par une voix humaine, vibrant dans l'air physique, adressés d'un corps à d'autres corps. Il y a une force à l'intérieur d'elles qui va vers l'extérieur d'elles, une force d'expression qui est aussi force d'expansion. Et puis, je voudrais encore ajouter ceci, en quoi s'énonce une reconnaissance : la lecture de Claude Simon n'invite pas seulement à la voix, elle est de celles, rares, qui suscitent, en une jalousie non mesquine, le désir d'écrire. Telle est la générosité de la beauté. "Toute belle œuvre ", note Barthes dans sa Préparation du Roman, "fonctionne comme une ouvre désirée, mais incomplète et comme perdue, parce que je ne l'ai pas faite moi-même, et qu'il faut la retrouver en la refaisant ; écrire c'est vouloir réécrire : je veux majouter activement à ce qui est beau et cependant me manque, me faut." L'œuvre de Claude Simon - et si loin que je m'en trouve, ça n'est 
pas la question -, ce grand souffle, grand arroi de mots, grand pneuma matériel, ce grand poème moderne en quoi résonnent les échos des littératures anciennes (parce que peu d'œuvres où se manifeste avec cette hauteur l'antiquité de la littérature), au sens où Barthes le dit, elle me faut. 